\title{
Airway Management of Penetrating Facial Trauma from an Impaled Screwdriver: A Case Report
}

\author{
Kelvin Wang BSE, Krishna B Shah and Suman Rajagopalan* \\ Department of Anesthesiology, Baylor College of Medicine, USA \\ *Corresponding author: Suman Rajagopalan MD, One Baylor Plaza, MS: BCM120, Houston, Texas, USA
}

\begin{abstract}
Airway management of a patient with penetrating facial trauma is often anticipated to be difficult. However, due to the variation in presentation of penetrating facial trauma, there are no consensus guidelines established for securing the airway. We present a case of penetrating facial trauma with an impaled screwdriver, describe the anatomical significance of the embedded foreign object, and suggest an approach to airway management. Ultimately, a skilled provider must use his or her clinical judgement and have a clear understanding of anatomy to best approach these unique scenarios.
\end{abstract}

\section{Introduction}

Penetrating facial trauma can present without a compromised airway, but some cases present major challenges with disruption of airway anatomy. Hemorrhage and soft tissue edema may lead to airway obstruction. Nasal bleeding can obscure the airway and the laryngeal and/or tracheal swelling can displace the epiglottis, arytenoid cartilages, and vocal cords, making for difficult visualization and increasing the risk of airway compromise. Other major concerns in these airways are the other potentially unknown injuries to the cervicofacial vasculature, teeth, cervical spine, and brain tissue [1]. Bag-mask ventilation and endotracheal intubation are predicted to be challenging in a patient with maxillofacial trauma. Effective pre-oxygenation prolongs the time that the patient can tolerate apnea, which can be crucial for intubation of an anticipated difficult airway in these patients. Oral endotracheal intubation with rapid-sequence induction and in-line stabilization of the neck is the gold standard in general trauma patients [2]. However, there is no current consensus amongst the anesthesia community on airway management of penetrating maxillofacial injuries and much variability exists [2-5]. Here, we present a case of an impaled foreign body to the face and our approach to airway management of this patient. Written consent was obtained from the patient and a Health Information Portability and Accountability Act (HIPAA) authorization form was completed. This manuscript adheres to the applicable Case Report (CARE) guidelines for case reports.

\section{Case Description}

A middle-aged male with an unknown past medical history presented with an impaled screwdriver within the left maxilla immediately adjacent to the nose. The patient was agitated, confused, and intoxicated. No loss of consciousness or other bodily injuries were noted. A computerized tomography scan performed in the emergency room showed the screwdriver to be penetrating through the maxilla and entering the clivus with the tip in the prepontine cistern. The patient was transported directly to the operating room with a cervical spine collar in place for emergent intubation and control of nasal hemorrhage. He was breathing spontaneously, and the physical examination revealed multiple midface fractures, significant bloody secretions from the oronasopharynx, and a Mallampati class IV airway. The location of the screwdriver prohibited bag-mask ventilation, so the patient was pre-oxygenated with a nasal cannula at $10 \mathrm{~L}$-min-1 of oxygen. He was then premedicated with midazolam and fentanyl prior to any intubation attempts. The oropharynx was gently suctioned, and $4 \%$ lidocaine hurricane spray was administered. Because of the anticipated difficult airway, an awake fiberoptic intubation was 
performed. The image from the bronchoscope was significantly obscured by bloody secretions from the injury.

In addition, the patient was unable to cooperate for the awake fiberoptic intubation, as he was intoxicated and confused. Hence, the procedure was abandoned. A second effort was then attempted using a C-MAC® video laryngoscope (Karl Storz, Tuttlingen, Germany) with a size 3 blade. In-line stabilization was performed by the anesthesia team, preventing extension or flexion of the cervical spine during the intubation attempt. A small dose of propofol was given, but no paralytic was administered, and the patient was maintained on spontaneous respiration. Direct visualization of the cords with a Grade 1 view was obtained on the video laryngoscope screen, without significant secretions blocking the view. A size 8.0 endotracheal tube was passed a traumatically through the cords, the cuff was inflated, and the endotracheal tube was secured (Figure 1). There were no desaturation episodes and oxygen saturation were maintained above 95\% throughout the procedure. Appropriate placement was confirmed by bilateral chest rise, equal and bilateral breath sounds, and positive end-tidal $\mathrm{CO}_{2}$ on capnography. Following intubation, the surgical team packed the nose to control the bleeding. The nasal ala and the vestibular lacerations were repaired, and the patient was transferred to interventional radiology for angiography and embolization of the bleeding vessels. Intraoperative fluoroscopy and angiography of the cerebral vessels revealed the final point of contact of the screwdriver to be the posterior face of the clivus, but without any significant cervical or intracranial vascular abnormalities (Figures 2\&3). The left sphenopalatine and descending palatine branches of the left maxillary artery were successfully embolized and the patient returned to the operating room for the removal of the screwdriver. The patient eventually underwent definitive surgical management of his facial fractures and was discharged home without complications.

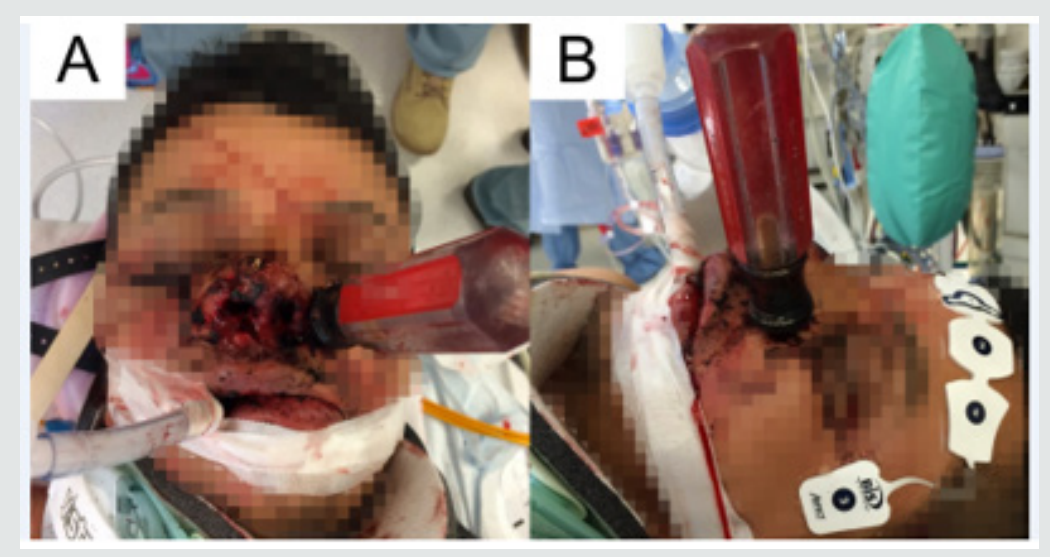

Figure 1: Frontal (A) and lateral (B) views after successful endotracheal intubation in a patient with a penetrating screwdriver injury using a C-MAC® video laryngoscope.

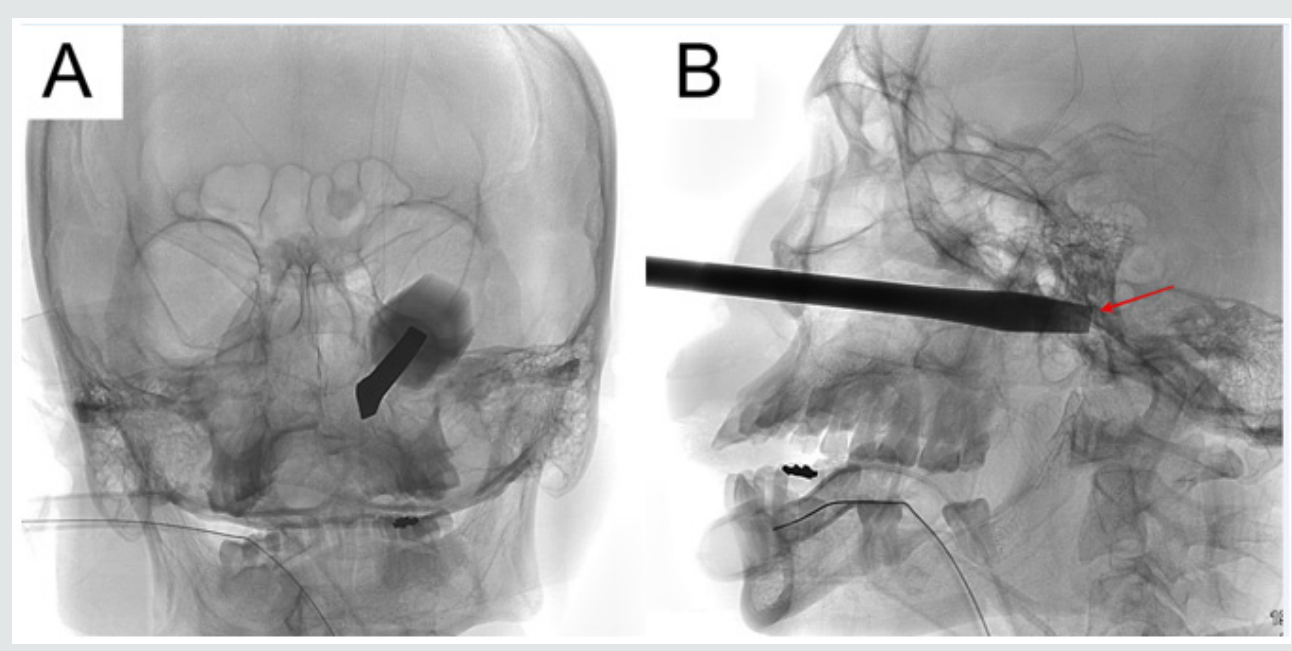

Figure 2: Intraoperative fluoroscopy showing the frontal (A) and lateral (B) views of the distal tip of the screwdriver embedded $1 \mathrm{~mm}$ into the clivus (red arrow). 


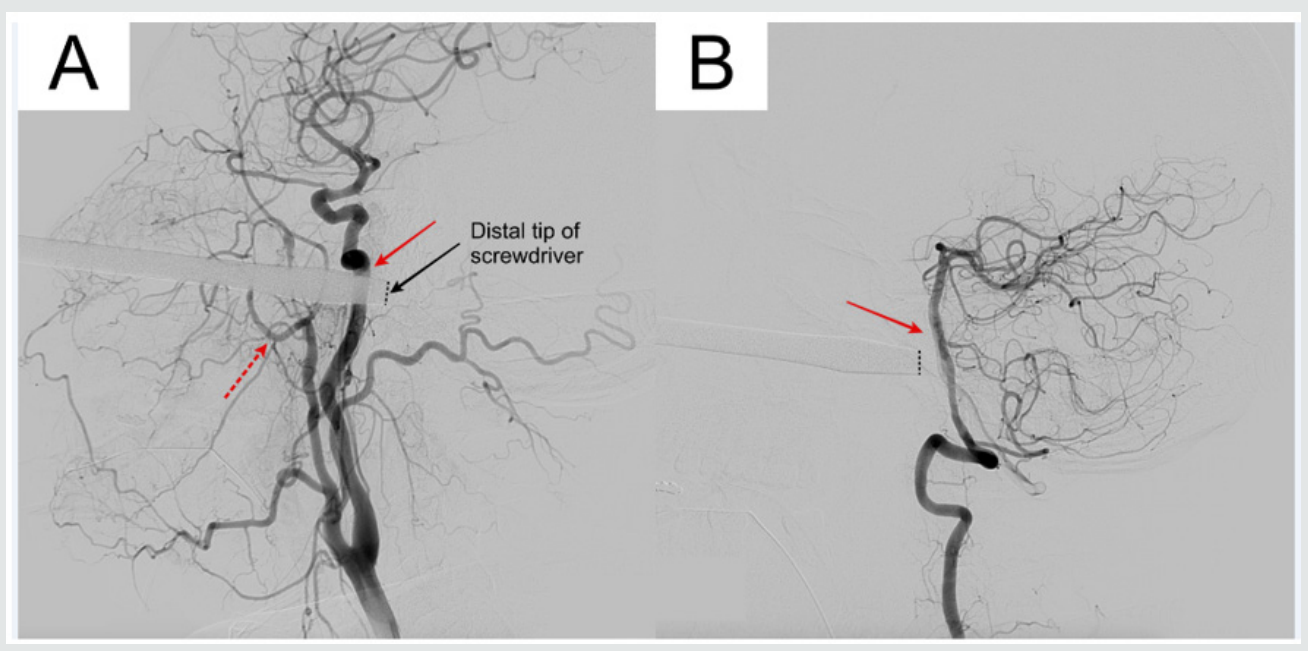

Figure 3: Cerebral angiogram showing proximity of the screwdriver to the left internal carotid artery (A, solid red arrow) and left basilar artery (B, solid red arrow). The patient's left maxillary artery (A, dotted red arrow) appeared intact.

\section{Discussion}

This patient had a screwdriver penetrating the clivus, a bony depression of the cranium that is occupied by the pons (Figure 2). The clivus is an important landmark because the basilar artery lies posterior to it; lateral to the clivus are the foramina lacerum bilaterally, from where the internal carotid arteries enter into the middle cranial fossa to form the Circle of Willis. Injury to any of these structures would likely have caused neurological injury or life-threatening bleeding. From the initial scan in the emergency room, it was unclear whether the screwdriver perforated any of the major blood vessel of the brain. The surgery team did not want to remove or move the screwdriver due to the risk of neurological injury. Thus, four vessel angiographies of the brain were performed to assess the integrity of blood vessels, and only when it was confirmed that no vessels were involved, was the screwdriver removed. In penetrating facial trauma, oropharyngeal anatomy could be distorted by edema or blocked by hemorrhage, which could prevent a proper mask fit and seal or effective air transfer to the lungs [2]. Facemask ventilation was impossible in this case due to the proximity of the screwdriver to the nose. Another concern at the time of intubation was that the tip of the screwdriver was known to be in the clivus, which meant any movement of the screwdriver by a mask pushing on it may result in vascular or brain damage. An aggravated patient who has impending hypoxemia combined with the risk of aspiration or cervical-spine injury could lead to the life-threatening "can't intubate, can't ventilate" scenario, especially if there is administration of a paralytic before securing the airway. In this case, we fitted a nasal cannula with high flow oxygen for pre-oxygenation to maximize oxygenation and held paralytics to allow the patient to breathe spontaneously during any intubation attempts as mask ventilation was difficult in this patient.
This patient also had significant bleeding into the oropharynx from the transection of the left sphenopalatine and descending palatine branches of the left maxillary artery, which branches from the left external carotid artery (Figure 3). Bleeding in the airway can contribute to difficult intubation and mask ventilation. A rapidly expanding hematoma causing airway obstruction must also be considered [6]. Even though awake fiberoptic intubation is the standard for traumatic airway injury and has been successfully performed in other instances of penetrating facial injury [5], it was unsuccessful in this case because the patient had active hemorrhage from an arterial transection that significantly obscured visualization of the airway anatomy. Furthermore, the patient was intoxicated and uncooperative with the fiberoptic intubation and every attempt with the bronchoscope only worsened his agitation. In emergency situations such as this, specialized experience plays a major role. The American Society of Anesthesiologists has a difficult airway algorithm, which has been adopted for trauma. As a part of the algorithm, if awake intubation is not possible and if the patient has a recognized difficult airway, then the next step in management suggests proceeding with surgical or percutaneous surgical access of the airway [7] Fortunately, while preparing for surgical airway access in this patient, the attempt with the C-MAC $®$ video laryngoscope was successful. The camera attached below the video laryngoscope blade was not blurred by blood, and successful endotracheal intubation was achieved while the patient was still breathing spontaneously. In addition, extreme caution was undertaken to avoid overextension of the neck or bucking, as the slightest movement could worsen a cervical spine injury or damage the basilar or internal carotid artery (Figure 3).

\section{Conclusion}

In conclusion, there is no unified approach for airway management in penetrating facial trauma. An appropriately 
skilled provider is needed to manage these airways and anticipate difficulties such as the inability to mask ventilate, severe hemorrhage, or obscured airway view. An anesthesiologist should be comfortable basing his or her decision-making process on the clinical scenario rather than relying on a preset algorithm. Finally, a full understanding of anatomy is required to consider the significance of other potentially unknown injuries to sensibly apply basic and advanced airway management techniques.

\section{Financial Disclosures}

None

\section{Conflicts of Interest}

None

\section{Author's Individual Contributions}

Kelvin Wang: This author helped prepare, write, and edit the manuscript.

Krishna B. Shah: This author helped prepare, write, and edit the manuscript.
Suman Rajagopalan: This author helped prepare, write, and edit the manuscript.

\section{References}

1. Hutchinson I, Lawlor M, Skinner D (1990) ABC of major trauma. Major maxillofacial injuries. BMJ 301(6752): 595-599.

2. Cogbill TH, Cothren CC, Ahearn MK, Daniel C Cullinane, Krista L Kaups et al. (2008) Management of maxillofacial injuries with severe oronasal hemorrhage: A multicenter perspective. J Trauma 65(5): 994-999.

3. Barak M, Bahouth H, Leiser Y, El-Naaj IA (2015) Airway management of the patient with maxillofacial trauma: Review of the literature and suggested clinical approach. Biomed Res Int 724032.

4. Mercer SJ, Lewis SE, Wilson SJ, Groom P, Mahoney PF (2010) Creating airway management guidelines for casualties with penetrating airway injuries. J R Army Med Corps 156: S355-360.

5. Joly LM, Oswald AM, Disdet M, Raggueneau JL (2002) Difficult endotracheal intubation as a result of penetrating cranio-facial injury by an arrow. Anesth Analg 94(1): 231-232.

6. Kotsev S (2005) Airway Management in a patient with a vascular injury and rapidly expanding neck hematoma. Eur J Anaesthesiol 22(7): 556558.

7. Apfelbaum JL, Hagberg CA, Caplan RA, et al. (2013) Practice guidelines for management of the difficult airway: An updated report by the American Society of Anesthesiologists Task Force on Management of the Difficult Airway. Anesthesiology 118(2): 251-270.

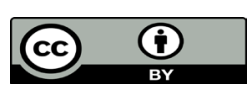

This work is licensed under Creative Commons Attribution 4.0 License

To Submit Your Article Click Here: Submit Article

DOI: $10.32474 /$ GJAPM.2020.03.000173

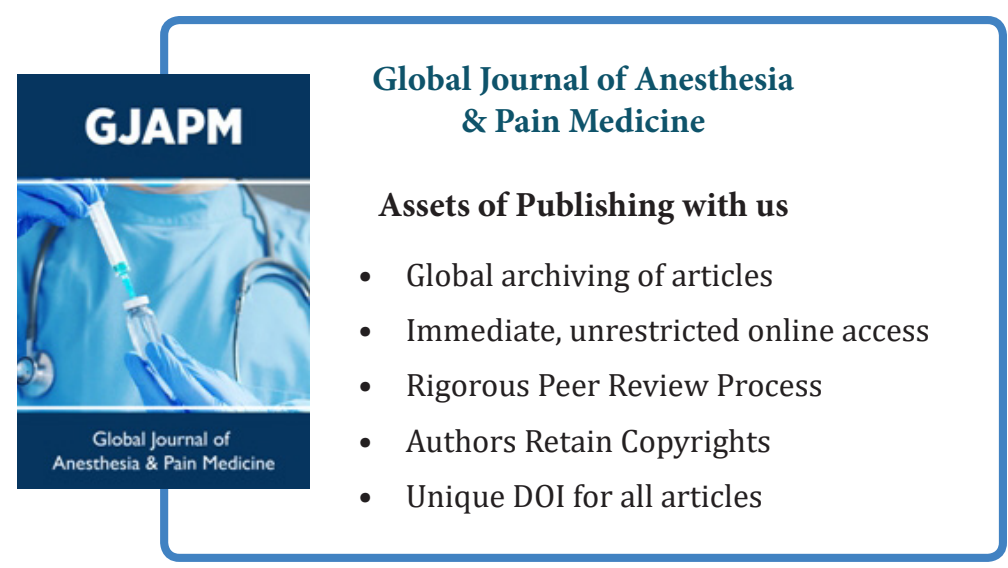

